\title{
Output Reachable Set Analysis for Periodic Positive Systems
}

\author{
Ilyas Sitli $\mathbb{D},{ }^{1}$ Fouad Lahmidi $\mathbb{D},{ }^{1}$ Abdelwahed Namir $\mathbb{D}^{2},{ }^{2}$ and Mouhcine Naim $\mathbb{D}^{1}$ \\ ${ }^{1}$ Laboratory of Analysis, Modeling and Simulation, Faculty of Sciences Ben M'sik, Hassan II University, Casablanca, Morocco \\ ${ }^{2}$ Laboratory of Information Technology and Modeling, Faculty of Sciences Ben M'sik, Hassan II University, Casablanca, Morocco
}

Correspondence should be addressed to Ilyas Sitli; sitli.ilyass@gmail.com

Received 25 September 2021; Accepted 22 October 2021; Published 15 November 2021

Academic Editor: Hernando Quevedo

Copyright (c) 2021 Ilyas Sitli et al. This is an open access article distributed under the Creative Commons Attribution License, which permits unrestricted use, distribution, and reproduction in any medium, provided the original work is properly cited.

In this study, we use the union of the bounding hyperpyramids to estimate the output reachable set for periodic positive systems under two classes of exogenous disturbances. Optimization algorithms are used to obtain the smallest bounding hyperpyramids possible. Finally, numerical examples are given to verify the theoretical results.

\section{Introduction}

In recent decades, periodic systems, whose parameters contain periodic properties, have received a lot of attention. The study of periodic systems is motivated by the fact that many real-world practical systems possess periodic characteristics and can thus be described as periodic systems. For example, a pendulum system is a periodic system with cyclic behavior [1]. In the literature, a lot of important results on linear periodic systems have been reported, see, e.g., [2-10].

On the contrary, several dynamical systems indicate that the state and output variables are forced to be positive, or at least nonnegative, at all times when initial conditions and inputs are nonnegative. This type of system is known in the literature as the positive system. Positive systems have many fields of application: chemistry, biology, sociology, and economics. Many important properties and applications of positive systems can be found in the works of Luenberger [11], Farina and Rinaldi [12], and Kaczorek [13].

Recently, dynamical systems with both periodic and positive properties have attracted the interest of many researchers. The stability and stabilization problems of discrete-time periodic positive systems were studied by Bougatef et al. [14] and Ait Rami and Napp [15]. For periodic positive systems with time delays, the stability problem was addressed in [16]. See also [17], for more periodic systems' research results.
Reachability, as a fundamental concept in control theory, has received a lot of attention. Numerous authors have investigated the reachability of positive systems for both discrete and continuous systems [18-23]. The set containing all system outputs that are reachable from the origin under a prescribed set of inputs is called the output reachable set. Many researchers have focused on characterizing the outputs' reachable sets for dynamical systems, but when the input signal is constrained, transferring the output of the system to an arbitrary desired output from the origin is generally difficult, so the popular technique in the literature is to determine a region as small as possible to bind the output reachable set. A usual strategy is to estimate the output reachable set by a few ellipsoids that can be determined by solving linear matrix inequality (LMI). Reachable set estimation problem has been studied for time-delay systems [24, 25], singular systems [26], periodic systems [27], positive systems [28], switched positive systems [29], etc.

This paper aims to solve the output reachable set estimation problem for periodic positive systems under two possible classes of nonnegative exogenous disturbances based on two norms.

The organization of this paper is the following form. In Section 2, the formulation of the problem is given and the positivity of the considered system is defined. In Section 3, results on the estimation of the output reachable set are given under two classes of exogenous disturbances, and the 
optimization techniques are used to obtain the smallest possible hyperpyramids which bound the output reachable set. Some examples are considered to verify the theoretical results.

Notations. The notations used in this work are $\mathbb{Z}_{+}$set of nonnegative integers, $\mathbb{Z}_{+}^{0}$ set of positive integers, $\mathbb{R}$ set of real numbers, $\mathbb{R}^{n}$ set of $n$-dimensional real vectors, $\mathbb{R}^{m \times n}$ set of $m \times n$ real matrices, $\mathbb{R}_{+}^{n}$ positive orthant of $\mathbb{R}^{n}, \overline{\mathbb{R}}_{+}^{n}$ closed positive orthant of $\mathbb{R}^{n}, A^{T}$ transpose of $A, 1$ vector $[1,1, \ldots, 1]^{T}, I$ identity matrix, $x \geq 0(x>0)$ every component of the vector $x$ is nonnegative (positive), $A \geq 0(A>0)$ every entry of matrix $A$ is nonnegative (positive), and $\sigma_{s}^{k}=$ $\{s, s+1, \ldots, k\}$ is the finite subset of $\mathbb{Z}_{+}$with $s \leq k$.

\section{Preliminaries}

Consider the discrete-time periodic linear system described by

$$
\begin{aligned}
& \left\{\begin{array}{l}
x_{t+1}=A_{t} x_{t}+B_{t} \vartheta_{t}, \quad t \in \mathbb{Z}_{+}, \\
x_{0} \in \mathbb{R}^{n},
\end{array}\right. \\
& y_{t}=C_{t} x_{t}, \quad t \in \mathbb{Z}_{+},
\end{aligned}
$$

where $x_{t} \in \mathbb{R}^{n}$ is the state vector, $\vartheta_{t} \in \mathbb{R}^{m}$ is the exogenous input signal, $y_{t} \in \mathbb{R}^{r}$ is the output vector, and $A_{t}, B_{t}$, and $C_{t}$ are real matrices with appropriate dimensions and we assume that there exists $T \in \mathbb{Z}_{+}^{0}$ such that, for all $t \in \mathbb{Z}_{+}$, we have $A_{t}=A_{t+T}, \quad B_{t}=B_{t+T}, \quad$ and $C_{t}=C_{t+T}$. We note $x:=\left(x_{t}\right)_{t \geq 0}, y:=\left(y_{t}\right)_{t \geq 0}$, and $\vartheta:=\left(\vartheta_{t}\right)_{t \geq 0}$.

Definition 1. Systems (1)-(2) are said to be positive if, for any initial condition $x_{0} \in \mathbb{R}_{+}^{n}$ and for any exogenous input $\vartheta_{t} \in \mathbb{R}_{+}^{m}$ and $t \in \mathbb{Z}_{+}$, we have $x_{t} \in \mathbb{R}_{+}^{n}$ and $y_{t} \in \mathbb{R}_{+}^{r}$, for all $t \in \mathbb{Z}_{+}$.

Remark 1. Systems (1)-(2) are positive if $A_{t} \geq 0, B_{t} \geq 0$, and $C_{t} \geq 0$, for all $t \in \sigma_{0}^{T-1}$.

In the rest of this paper, we assume that systems (1)-(2) are positive.

The results presented in this paper are divided into two cases according to the following norms: $\|\vartheta\|_{1,1}=\sum_{t=0}^{\infty}\left\|\vartheta_{t}\right\|_{1}$ and $\|\vartheta\|_{\infty, 1}=\sup _{t \geq 0}\left\|\vartheta_{t}\right\|_{1}$, where $\left\|\vartheta_{t}\right\|_{1}=\sum_{i=0}^{m}\left|\vartheta_{t, i}\right|$, for $\vartheta_{t}=\left[\vartheta_{t, 1}, \vartheta_{t, 2}, \ldots, \vartheta_{t, m}\right]^{T} \in \mathbb{R}^{m}$.

Case $\quad 1: \quad \vartheta \in \Sigma_{1,1}^{+}:=\left\{\vartheta \mid\|\vartheta\|_{1,1} \leq 1\right.$ and $\vartheta_{t} \geq 0$, for all $t \in$ $\left.\mathbb{Z}_{+}\right\}$.

The output reachable set in this case is defined as follows:

$$
\mathfrak{R}_{1,1}^{+}:=\left\{y \mid \text { there is } \vartheta \in \Sigma_{1,1}^{+} \text {such that } y \text { and } \vartheta \text { satisfy }(1)-(2) \text { with } x_{0}=0\right\} \text {. }
$$

Case 2: $\vartheta \in \Sigma_{\infty, 1}^{+}:=\left\{\vartheta \mid\|\vartheta\|_{\infty, 1} \leq 1\right.$ and $\vartheta_{t} \geq 0$, for all $t \in$ $\left.\mathbb{Z}_{+}\right\}$.
The output reachable set in this case is defined as follows:

$$
\mathfrak{R}_{\infty, 1}^{+}:=\left\{y \mid \text { there is } \vartheta \in \Sigma_{\infty, 1}^{+} \text {such that } y \text { and } \vartheta \text { satisfy }(1)-(2) \text { with } x_{0}=0\right\} \text {. }
$$

The output reachable set in this paper will be bounded by hyperpyramids of the form

$$
\mathfrak{I}(\xi):=\left\{\zeta \in \overline{\mathbb{R}}_{+}^{r} \mid \xi^{T} \zeta \leq 1\right\}, \quad \text { where } \xi \in \mathbb{R}_{+}^{r} .
$$

\section{Main Results}

In this section, we will estimate the output reachable set of systems (1)-(2) by hyperpyramids for the two cases mentioned above. For that, we will use optimization techniques to obtain the smallest possible hyperpyramids.

\subsection{Estimation of Output Reachable Sets}

3.1.1. Case 1. In this case, we consider systems (1)-(2) under zero initial conditions and exogenous input $\vartheta \in \Sigma_{1,1}^{+}$.
Lemma 1. Let $\varphi_{t}: \mathbb{R}_{+}^{r} \longrightarrow \mathbb{R}_{+}, t \geq 0$, be a set of functions such that

(i) $\varphi_{0}(0)=0$.

(ii) If $y \in \mathfrak{R}_{1,1}^{+}$and $\vartheta \in \Sigma_{1,1}^{+}$is the corresponding input, we have

$$
\varphi_{t+1}\left(y_{t+1}\right)-\varphi_{t}\left(y_{t}\right) \leq 1^{T} \vartheta_{t}, \quad \forall t \in \mathbb{Z}_{+} .
$$

Then, we obtain $\varphi_{t}\left(y_{t}\right) \leq 1, \forall y \in \mathfrak{R}_{1,1}^{+}$and $\forall t \in \mathbb{Z}_{+}$.

Proof. Let $y \in \mathfrak{R}_{1,1}^{+}$and $\vartheta \in \Sigma_{1,1}^{+}$be the corresponding input. Then, $\forall t \in \mathbb{Z}_{+}^{0}$, and we have

$$
\begin{gathered}
\varphi_{t}\left(y_{t}\right)-\varphi_{t-1}\left(y_{t-1}\right) \leq 1^{T} \vartheta_{t-1}=\left\|\vartheta_{t-1}\right\|_{1}, \\
\vdots \\
\varphi_{1}\left(y_{1}\right)-\varphi_{0}\left(y_{0}\right) \leq 1^{T} \vartheta_{0}=\left\|\vartheta_{0}\right\|_{1} .
\end{gathered}
$$


So, we obtain

$$
\varphi_{t}\left(y_{t}\right)-\varphi_{0}\left(y_{0}\right) \leq \sum_{s=0}^{t-1} 1^{T} \vartheta_{s} \leq\|\vartheta\|_{1,1} \leq 1 .
$$

Since $\varphi_{0}\left(y_{0}\right)=\varphi_{0}(0)=0$, then we get $\varphi_{t}\left(y_{t}\right) \leq 1$. The proof is completed.

According to this lemma, we get the following bounding of the output reachable set.

Theorem 1. Consider systems (1)-(2), and assume that there exist $\xi_{l} \in \mathbb{R}_{+}^{r}, l \in \sigma_{0}^{T}$, with $\xi_{T}=\xi_{0}$, such that, for all $l \in \sigma_{0}^{T-1}$,

$$
\left\{\begin{array}{l}
A_{l}^{T} C_{l+1}^{T} \xi_{l+1}-C_{l}^{T} \xi_{l} \leq 0, \\
B_{l}^{T} C_{l+1}^{T} \xi_{l+1} \leq 1
\end{array}\right.
$$

Then, $\mathfrak{R}_{1,1}^{+} \subset \cup_{l=0}^{T-1} \mathfrak{\Im}\left(\xi_{l}\right)$ (i.e., the output reachable set is bounded by the union of a set of hyperpyramids).

Proof. Let $\left(\xi_{t}\right)_{t \geq 0}$ be the sequence defined by $\xi_{t}=\xi_{t+T}$, $\forall t \in \mathbb{Z}_{+}$, and let, for $t \geq 0$,

$$
\begin{array}{r}
\varphi_{t}: \mathbb{R}_{+}^{r} \longrightarrow \mathbb{R}_{+}, \\
z \longmapsto \xi_{t}^{T} z .
\end{array}
$$

Let $y \in \mathfrak{R}_{1,1}^{+}$and $\vartheta \in \Sigma_{1,1}^{+}$be the corresponding input. For any $t \geq 0$, there is $l \in \sigma_{0}^{T-1}$ such that $t \equiv l[T]$. Then, we obtain

$$
\begin{aligned}
\varphi_{t+1}\left(y_{t+1}\right)-\varphi_{t}\left(y_{t}\right) & =\left(\xi_{t+1}^{T} C_{t+1} A_{t}-\xi_{t}^{T} C_{t}\right) x_{t}+\xi_{t+1}^{T} C_{t+1} B_{t} \vartheta_{t} \\
& =\left(\xi_{l+1}^{T} C_{l+1} A_{l}-\xi_{l}^{T} C_{l}\right) x_{t}+\xi_{l+1}^{T} C_{l+1} B_{l} \vartheta_{t} \\
& \leq 1^{T} \vartheta_{t} .
\end{aligned}
$$

According to Lemma 1, we get $\varphi_{t}\left(y_{t}\right) \leq 1$. And, the periodicity of $\xi_{t}$ implies that $y_{t} \in \cup_{l=0}^{T-1} \mathfrak{I}\left(\xi_{l}\right)$. So, $\mathfrak{R}_{1,1}^{+} \subset \cup_{l=0}^{T-1} \mathfrak{\Im}\left(\xi_{l}\right)$. The proof is completed.

If $T=1$, then systems (1)-(2) reduce to the linear positive time-invariant system given by

$$
\begin{aligned}
& \left\{\begin{array}{l}
x_{t+1}=A x_{t}+B \vartheta_{t}, \quad t \in \mathbb{Z}_{+}, \\
x_{0} \in \mathbb{R}^{n} .
\end{array}\right. \\
& y_{t}=C x_{t}, \quad t \in \mathbb{Z}_{+} .
\end{aligned}
$$

From Theorem 1, we can deduce the following corollary to determine an estimation of the output reachable set of systems (12)-(13).

Corollary 1. Consider systems (12)-(13), and assume that there exists $\xi \in \mathbb{R}_{+}^{r}$ such that the following condition holds:

$$
\left\{\begin{array}{l}
A^{T} C^{T} \xi-C^{T} \xi \leq 0, \\
B^{T} C^{T} \xi \leq 1 .
\end{array}\right.
$$

Then, $\mathfrak{R}_{1,1}^{+} \subset \mathfrak{J}(\xi)$.
3.1.2. Case 2. In this case, we consider systems (1)-(2) under zero initial conditions and exogenous input $\vartheta \in \Sigma_{\infty, 1}^{+}$.

Lemma 2. Let $\varphi_{t}: \mathbb{R}_{+}^{r} \longrightarrow \mathbb{R}_{+}, t \in \mathbb{Z}_{+}$, be a set of functions such that

(i) $\varphi_{0}(0)=0$.

(ii) There exist $\theta_{t} \in[0,1], t \in \mathbb{Z}_{+}$, such that if $y \in \mathfrak{R}_{\infty, 1}^{+}$ and $\vartheta \in \Sigma_{\infty, 1}^{+}$is the corresponding input, then

$$
\varphi_{t+1}\left(y_{t+1}\right)-\theta_{t} \varphi_{t}\left(y_{t}\right) \leq\left(1-\theta_{t}\right) 1^{T} \vartheta_{t}, \quad \forall t \in \mathbb{Z}_{+} .
$$

Then, we obtain $\varphi_{t}\left(y_{t}\right) \leq 1, \forall t \in \mathbb{Z}_{+}$.

Proof. Let $y \in \mathfrak{R}_{\infty, 1}^{+}$and $\vartheta \in \Sigma_{\infty, 1}^{+}$be the corresponding input, and we have

$$
\varphi_{t+1}\left(y_{t+1}\right)-\theta_{t} \varphi_{t}\left(y_{t}\right) \leq\left(1-\theta_{t}\right) 1^{T} \vartheta_{t} \leq\left(1-\theta_{t}\right)\|\vartheta\|_{\infty, 1} \leq 1-\theta_{t} .
$$

Then, $\varphi_{t+1}\left(y_{t+1}\right)-1 \leq \theta_{t}\left(\varphi_{t}\left(y_{t}\right)-1\right)$.

For any time $t \in \mathbb{Z}_{+}^{0}$, we have

$$
\begin{aligned}
\varphi_{t}\left(y_{t}\right)-1 \leq & \theta_{t-1}\left(\varphi_{t-1}\left(y_{t-1}\right)-1\right) \\
& \leq \theta_{t-1} \theta_{t-2}\left(\varphi_{t-2}\left(y_{t-2}\right)-1\right) \\
& \vdots \\
& \leq \theta_{t-1} \cdots \theta_{0}\left(\varphi_{0}\left(y_{0}\right)-1\right) .
\end{aligned}
$$

Since $\varphi_{0}\left(y_{0}\right)=\varphi_{0}(0)=0 \leq 1$, it follows that $\varphi_{t}\left(y_{t}\right) \leq 1$. The proof is completed.

Using this lemma, we can derive the following bounding of the output reachable set.

Theorem 2. Consider systems (1)-(2), and assume that there exist $\xi_{l} \in \mathbb{R}_{+}^{r}, l \in \sigma_{0}^{T}$, with $\xi_{T}=\xi_{0}$ and $\theta_{l} \in[0,1], l \in \sigma_{0}^{T-1}$, such that, for all $l \in \sigma_{0}^{T-1}$, we have

$$
\left\{\begin{array}{l}
A_{l}^{T} C_{l+1}^{T} \xi_{l+1}-\theta_{l} C_{l}^{T} \xi_{l} \leq 0, \\
B_{l}^{T} C_{l+1}^{T} \xi_{l+1} \leq\left(1-\theta_{l}\right) 1 .
\end{array}\right.
$$

Then, $\mathfrak{R}_{\infty, 1}^{+} \subset \cup_{l=0}^{T-1} \mathfrak{\Im}\left(\xi_{l}\right)$.

Proof. We construct two sequences $\left(\xi_{t}\right)_{t \geq 0}$ and $\left(\theta_{t}\right)_{t \geq 0}$ by posing $\xi_{t}=\xi_{t+T}$ and $\theta_{t}=\theta_{t+T}, \forall t \in \mathbb{Z}_{+}$. We consider the family of functions:

$$
\begin{array}{r}
\varphi_{t}: \mathbb{R}_{+}^{r} \longrightarrow \mathbb{R}_{+}, \\
z \longmapsto \xi_{t}^{T} z .
\end{array}
$$

Let $y \in \mathfrak{R}_{\infty, 1}^{+}$and $\vartheta \in \Sigma_{\infty, 1}^{+}$be the corresponding input. For all $t \in \mathbb{Z}_{+}$, there is $l \in \sigma_{0}^{T-1}$ such that $t \equiv l[T]$. Then, we obtain 


$$
\begin{aligned}
\varphi_{t+1}\left(y_{t+1}\right)-\theta_{t} \varphi_{t}\left(y_{t}\right) & =\left(\xi_{t+1}^{T} C_{t+1} A_{t}-\theta_{t} \xi_{t}^{T} C_{t}\right) x_{t}+\xi_{t+1}^{T} C_{t+1} B_{t} \vartheta_{t} \\
& =\left(\xi_{l+1}^{T} C_{l+1} A_{l}-\theta_{l} \xi_{l}^{T} C_{l}\right) x_{t}+\xi_{l+1}^{T} C_{l+1} B_{l} \vartheta_{t} \\
& \leq\left(1-\theta_{l}\right) 1^{T} \vartheta_{t} \\
& =\left(1-\theta_{t}\right) 1^{T} \vartheta_{t} .
\end{aligned}
$$

So, Lemma 2 implies that $\varphi_{t}\left(y_{t}\right) \leq 1$, and from the periodicity of $\xi_{t}$, we obtain $y_{t} \in \cup_{l=0}^{T-1} \mathfrak{\Im}\left(\xi_{l}\right)$. So, $\mathfrak{R}_{\infty, 1}^{+} \subset \cup_{l=0}^{T-1} \mathfrak{I}\left(\xi_{l}\right)$. The proof is completed.

If $T=1$, we can deduce the following corollary for the estimation of the output reachable set of the linear positive time-invariant systems (12)-(13).

Corollary 2. Consider systems (12)-(13), and assume that there exists $\xi \in \mathbb{R}_{+}^{r}$ such that the following condition holds:

$$
\left\{\begin{array}{l}
A^{T} C^{T} \xi-\theta C^{T} \xi \leq 0, \\
B^{T} C^{T} \xi \leq(1-\theta) 1 .
\end{array}\right.
$$

Then, $\mathfrak{R}_{\infty, 1}^{+} \subset \mathfrak{I}(\xi)$.

Remark 2. In Theorem 1 (Theorem 2), the norms of the exogenous inputs are no greater than 1 . We can deduce the estimation of the output reachable set if the norms of the exogenous inputs are no greater than a scalar $\widehat{\vartheta}>0$. If $\|\vartheta\|_{1,1} \leq \widehat{\vartheta}\left(\|\vartheta\|_{\infty, 1} \leq \widehat{\vartheta}\right)$, then the output reachable set $\mathfrak{R}_{1,1}^{+}\left(\mathfrak{R}_{\infty, 1}^{+}\right)$can be bounded by the union of a set of hyperpyramids $\cup_{l=0}^{T-1} \mathfrak{\Im}\left(\xi_{l} / \widehat{\vartheta}\right)$. Indeed, if we assume that $\|\vartheta\|_{1,1} \leq \widehat{\vartheta} \quad\left(\|\vartheta\|_{\infty, 1} \leq \hat{\vartheta}\right)$, then we obtain $\|(\vartheta / \hat{\vartheta})\|_{1,1} \leq 1\left(\|(\vartheta / \hat{\vartheta})\|_{\infty, 1} \leq 1\right)$. Systems (1)-(2) can be rewritten in the following form:

$$
\left\{\begin{array}{l}
\frac{x_{t+1}}{\widehat{\vartheta}}=A_{t} \frac{x_{t}}{\widehat{\vartheta}}+B_{t} \frac{\vartheta_{t}}{\widehat{\vartheta}}, \\
\frac{y_{t}}{\widehat{\vartheta}}=C_{t} \frac{x_{t}}{\widehat{\vartheta}}
\end{array}\right.
$$

So, according to Theorem 1 (Theorem 2), $y_{t} / \widehat{\vartheta}$ can be bounded by $\cup_{l=0}^{T-1} \mathfrak{\Im}\left(\xi_{l}\right)$. Then, $y_{t}$ can be bounded by $\cup_{l=0}^{T-1} \mathfrak{\Im}\left(\xi_{l} / \widehat{\vartheta}\right)$.

The hypervolume of the hyperpyramid (5) is equal to $1 /\left(r ! \prod_{i=1}^{r}\left(1 / \xi_{i}\right)\right)$ (Carter and Champanerkar [30]). The volume of the bounding hyperpyramids considered in the two cases can be minimised by solving the following optimization problem:

$$
\min \left(-\sum_{m=1}^{r} \sum_{l=0}^{T-1} \log \xi_{l, m}\right),
$$

which subjects to the conditions in Theorems 1 or 2, with $\xi_{l}=\left[\xi_{l, 1}, \xi_{l, 2}, \ldots, \xi_{l, r}\right]^{T}, l \in \sigma_{0}^{T-1}$.

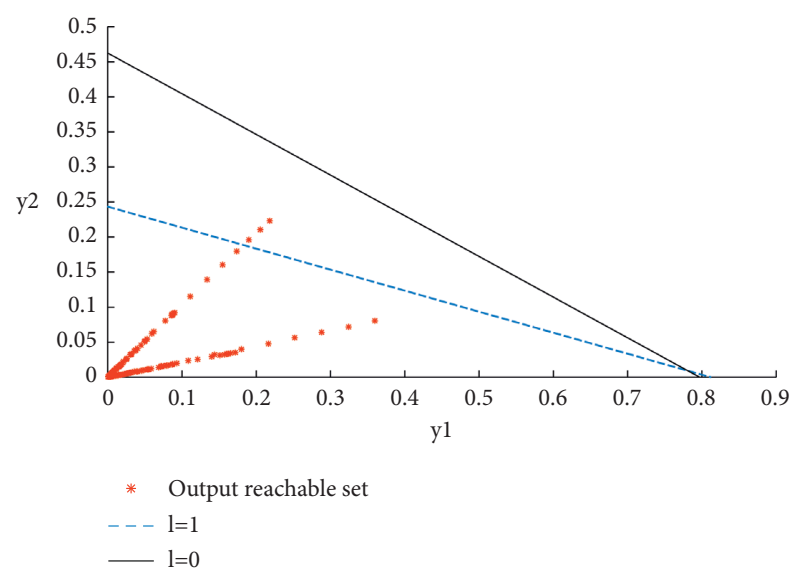

FIGURE 1: Estimation of output reachable set with $\vartheta \in \Sigma_{1,1}^{+}$.

To solve optimization problem (23) which subjects to the conditions in Theorem 2, we adopt genetic algorithm (GA). For more details about GA, see [31, 32].

3.2. Examples. In this section, we will give examples for the two cases.

3.2.1. Case 1. Consider systems (1)-(2) with period $T=2$, and

$$
\begin{aligned}
& \left(A_{0}, B_{0}, C_{0}\right)=\left(\left[\begin{array}{ll}
0.1 & 0.6 \\
0.2 & 0.4
\end{array}\right],\left[\begin{array}{l}
0.5 \\
0.3
\end{array}\right],\left[\begin{array}{ll}
0.3 & 0.7 \\
0.1 & 0.1
\end{array}\right]\right), \\
& \left(A_{1}, B_{1}, C_{1}\right)=\left(\left[\begin{array}{ll}
0.5 & 0.2 \\
0.4 & 0.2
\end{array}\right],\left[\begin{array}{l}
0.2 \\
0.1
\end{array}\right],\left[\begin{array}{ll}
0.2 & 0.6 \\
0.3 & 0.5
\end{array}\right]\right) .
\end{aligned}
$$

By solving problem (23), we obtain

$$
\begin{aligned}
& \xi_{0}=\left[\begin{array}{l}
1.2321 \\
4.1090
\end{array}\right], \\
& \xi_{1}=\left[\begin{array}{l}
1.2549 \\
2.1621
\end{array}\right] .
\end{aligned}
$$

The bounding hyperpyramids are shown in Figure 1 with exogenous inputs defined by $\vartheta_{t}=(1-m) \times m^{t}, m=$ $0,0.1, \ldots, 0.9$.

3.2.2. Case 2. Consider systems (1)-(2) with period $T=2$ and

$$
\begin{aligned}
& \left(A_{0}, B_{0}, C_{0}\right)=\left(\left[\begin{array}{ll}
0.5 & 0.2 \\
0.6 & 0.4
\end{array}\right],\left[\begin{array}{l}
0.5 \\
0.3
\end{array}\right],\left[\begin{array}{ll}
0.2 & 0.5 \\
0.1 & 0.2
\end{array}\right]\right), \\
& \left(A_{1}, B_{1}, C_{1}\right)=\left(\left[\begin{array}{ll}
0.7 & 0.1 \\
0.3 & 0.2
\end{array}\right],\left[\begin{array}{l}
0.1 \\
0.4
\end{array}\right],\left[\begin{array}{ll}
0.1 & 0.1 \\
0.4 & 0.6
\end{array}\right]\right) .
\end{aligned}
$$

The optimal values of problem (23) are 


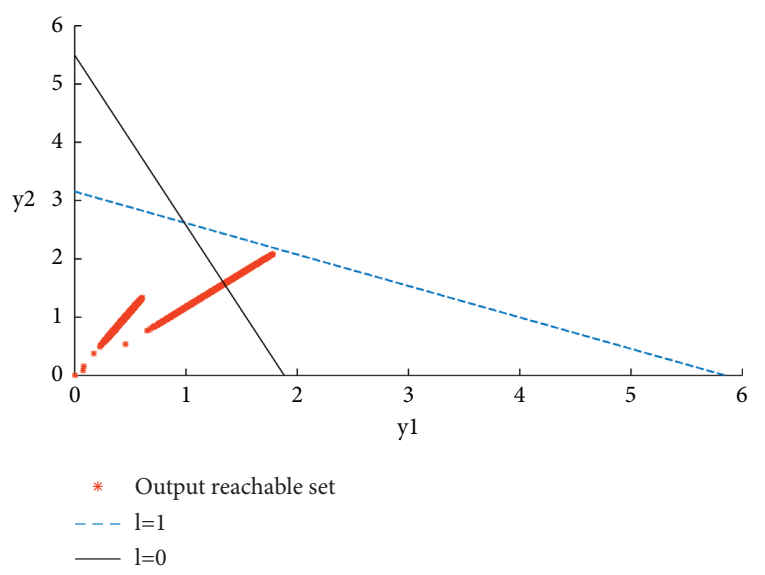

(a)

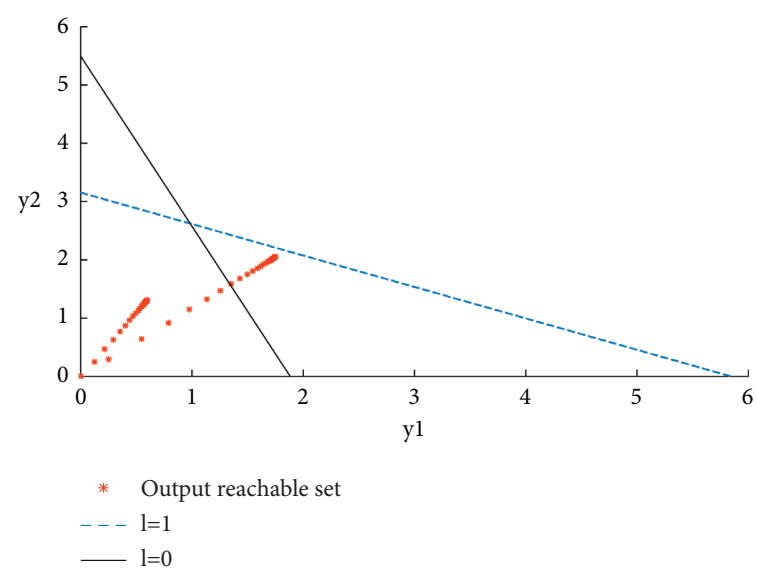

(b)

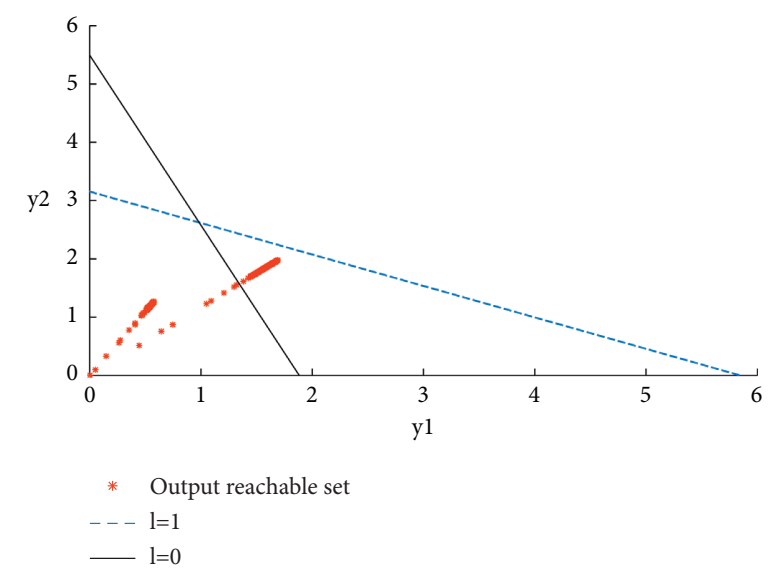

(c)

Figure 2: Estimation of the output reachable set with $\vartheta \in \Sigma_{\infty, 1}^{+}$.

$$
\begin{aligned}
& \theta_{0}=0.854, \\
& \theta_{1}=0.935, \\
& \xi_{0}=\left[\begin{array}{l}
0.171 \\
0.317
\end{array}\right], \\
& \xi_{1}=\left[\begin{array}{l}
0.531 \\
0.182
\end{array}\right] .
\end{aligned}
$$

The bounding hyperpyramids are shown in Figure 2 with exogenous inputs defined by $(a) \vartheta_{t}=\operatorname{rand}(t),(b) \vartheta_{t}=0.7$, and $(c) \vartheta_{t}=|\sin (t)|$.

\section{Conclusion}

In this work, we have studied the estimation of the output reachable set for positive periodic systems. Results (Theorem 1 and Theorem 2) have been found for two exogenous disturbance classes, and optimization techniques have been used to minimize the volume of bounding hyperpyramids. Numerical examples have been used to verify the theoretical results.

\section{Data Availability}

No data were used to support this study.

\section{Conflicts of Interest}

The authors declare that they have no conflicts of interest.

\section{References}

[1] S. Bittanti, D. B. Hernandez, and G. Zerbi, "The simple pendulum and the periodic LQG control problem," Journal of the Franklin Institute, vol. 328, no. 2, pp. 299-315, 1991.

[2] S. Bittanti, "Deterministic and stochastic linear periodic systems," in Time Series and Linear Systems, pp. 141-182, Springer-Verlag, Berlin, Germany, 1986.

[3] S. Bittanti, P. Bolzern, and P. Colaneri, "Stability analysis of linear periodic systems via the Lyapunov equation," in Proceedings of the 10th IFAC World Congress, pp. 169-172, Budapest, Hungary, 1984.

[4] S. Bittanti and P. Bolzern, "Discrete-time linear periodic systems: gramian and modal criteria for reachability and controllability†," International Journal of Control, vol. 41, no. 4, pp. 909-928, 1985. 
[5] S. Bittanti and P. Bolzern, "Stabilizability and detectability of linear periodic systems," Systems \& Control Letters, vol. 6, pp. 141-145, 1985.

[6] D. Arzelier, D. Peaucelle, and C. Farges, "Robust analysis and synthesis of linear polytopic discrete-time periodic systems via LMIs," in Proceedings of the 44th IEEE Conference on Decision and Control, pp. 5734-5739, Seville, Spain, December 2005.

[7] S. Bittanti, P. Colaneri, and G. De Nicolao, "The difference periodic Ricati equation for the periodic prediction problem," IEEE Transactions on Automatic Control, vol. 33, no. 8, pp. 706-712, 1988.

[8] P. Bolzern and P. Colaneri, "The periodic Lyapunov equation," SIAM Journal on Matrix Analysis and Applications, vol. 9, no. 4, pp. 499-512, 1988.

[9] B. Naima, "State feedback stabilization of a class of uncertain periodic systems," in Proceedings of the IFAC, vol. 44, pp. 12562-12567, Milano, Italy, 2011.

[10] C. E. De Souza and A. Trofino, "An LMI approach to stabilization of linear discrete-time periodic systems," International Journal of Control, vol. 73, no. 8, pp. 696-703, 2000.

[11] D. G. Luenberger, Introduction to Dynamic Systems: Theory, Models and Applications, Academic Press, New York, NY, USA, 1979.

[12] L. Farina and S. Rinaldi, Positive Linear Systems: Theory and Applications, J. Wiley \& Sons, New York, NY, USA, 2000.

[13] T. Kaczorek, Positive $1 D$ and $2 D$ systems, Communications and Control Engineering, Springer-Verlag, London, UK, 2002.

[14] N. Bougatef, M. Chaabane, O. Bachelier, D. Mehdi, and S. Cauet, "On the stabilization of a class of periodic positive discrete time systems," in Proceedings of the 49th IEEE Conference on Decision and Control (CDC), pp. 4311-4316, Atlanta, GA, USA, December 2010.

[15] M. Ait Rami and D. Napp, "Discrete-time positive periodic systems with state and control constraints," IEEE Transactions on Automatic Control, vol. 61, no. 1, pp. 234-239, 2016.

[16] X. Liu, "Stability analysis of periodic positive systems with delays," in Proceedings of the 31st Chinese Control Conference, pp. 95-100, Hefei, China, July 2012.

[17] S. Bittanti and P. Colaneri, Periodic Systems: Filtering and Control, Springer, London, UK, 2008.

[18] M. E. Valcher, "Controllability and reachability criteria for discrete time positive systems," International Journal of Control, vol. 65, no. 3, pp. 511-536, 1996.

[19] M. E. Valcher, "Reachability properties of continuous-time positive systems," IEEE Transactions on Automatic Control, vol. 54, no. 7, pp. 1586-1590, 2009.

[20] L. Caccetta and V. G. Rumchev, "A survey of reachability and controllability for positive linear systems," Annals of Operations Research, vol. 98, no. 1/4, pp. 101-122, 2000.

[21] Y. Ohta, H. Maeda, and S. Kodama, "Reachability, observability, and realizability of continuous-time positive systems," SIAM Journal on Control and Optimization, vol. 22, no. 2, pp. 171-180, 1984.

[22] Z. Bartosiewicz, "Local positive reachability of nonlinear continuous-time systems," IEEE Transactions on Automatic Control, vol. 61, no. 12, pp. 4217-4221, 2016.

[23] Z. Bartosiewicz, "Positive reachability of discrete-time nonlinear systems," in Proceedings of the 2016 IEEE Conference on Control Applications (CCA), pp. 1203-1208, IEEE, Buenos Aires, Argentina, September 2016.

[24] E. Fridman and U. Shaked, "On reachable sets for linear systems with delay and bounded peak inputs," Automatica, vol. 39, no. 11, pp. 2005-2010, 2003.
[25] P. T. Nam and P. N. Pathirana, "Further result on reachable set bounding for linear uncertain polytopic systems with interval time-varying delays," Automatica, vol. 47, no. 8, pp. 1838-1841, 2011.

[26] Z. Feng and J. Lam, "On reachable set estimation of singular systems,” Automatica, vol. 52, pp. 146-153, 2015.

[27] Y. Chen and J. Lam, "Estimation and synthesis of reachable set for discrete-time periodic systems," Optimal Control Applications and Methods, vol. 37, no. 5, pp. 885-901, 2015.

[28] B. Du, J. Lam, Z. Shu, and Y. Chen, "On reachable sets for positive linear systems under constrained exogenous inputs," Automatica, vol. 74, pp. 230-237, 2016.

[29] Y. Chen, J. Lam, J. Shen, B. Du, and P. Li, "Reachable set estimation for switched positive systems," International Journal of Systems Science, vol. 49, no. 11, pp. 2341-2352, 2018.

[30] J. S. Carter and A. Champanerkar, "A geometric method to compute some elementary integrals," 2008, https://arxiv.org/ pdf/math/0608722.pdf.

[31] M. Gen and R. Cheng, Genetic Algorithms and Engineering Optimization, John Wiley \& Sons, New York, NY, USA, 2000.

[32] G. Winter, J. Periaux, M. Galan, and P. Cuesta, Genetic Algorithms in Engineering and Computer Science, John Wiley \& Sons, New York, NY, USA, 1996. 